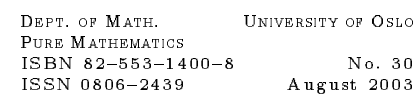

\title{
Div-Curl lemma for Edge Elements
}

\author{
Snorre H. Christiansen*
}

August 27, 2003

\begin{abstract}
A variant of Murat and Tartar's div-curl lemma is stated and proved for Nédélec's edge elements. For sequences of vector fields in sequences of such finite element spaces the hypothesis on the divergence can be put into a somewhat weaker form well-suited for the analysis of some numerical schemes. The proof uses a uniform norm equivalence related to discrete compactness properties of vector FE spaces and a super-approximation property of scalar FE spaces.
\end{abstract}

\section{Introduction}

Given two sequences of vector fields converging weakly in $\mathrm{L}^{2}$, the div-curl lemma of Murat [26] and Tartar [34], recalled in Theorem 1.1 underneath, gives sufficient conditions under which their scalar product converges to the right scalar field in the weak-star sense of distributions. Following Folland [18] I shall refer to weak-star as vague convergence, since this leads to adjectives more easily. The space of compactly supported smooth functions is denoted $\mathcal{D}$ and equipped with the topology relevant to the distribution theory of Schwartz.

Theorem 1.1 Suppose $\left(u_{h}\right)$ and $\left(u_{h}^{\prime}\right)$ are sequences of vector fields converging weakly in $\mathrm{L}^{2}$ to $u$ and $u^{\prime}$. Suppose furthermore that $\left(\operatorname{div} u_{h}\right)$ is relatively compact in $\mathrm{H}^{-1}$ and that ( $\operatorname{curl} u_{h}^{\prime}$ ) is relatively compact in $\mathrm{H}^{-1}$. Then $\left(u_{h} \cdot u_{h}^{\prime}\right)$ converges vaguely to $u \cdot u^{\prime}$ in $\mathcal{D}^{\prime}$.

Over the years the curl conforming FE spaces constructed by Nédélec [28][29], also referred to as edge elements since the principal degrees of freedom are attached to the edges, have emerged as the spaces of choice for discretizing Maxwell's equations both in frequency domain (see the recent book by Monk [25]) and time domain (see Joly [22]). The reasons for this success are slowly being uncovered. In particular it has been remarked by Costabel that for domains with reentrant corners, $\mathrm{H}^{1}$ conforming vector finite elements satisfying the perfect conductor boundary condition will fail to capture corner singularities of electromagnetic fields. It has also been shown by Boffi et al. that some tempting nodal FE spaces yield spurious eigenvalues when used to compute the eigenvalues of the curlcurl operator. Moreover it has been shown that edge elements suffer from none of these drawbacks.

${ }^{*}$ CMA c/o Matematisk Institutt, PB 1053 Blindern, N-0316 Oslo, Norway. email : snorrec@math .uio.no 
In this paper I intend to prove another good property of edge elements, this time related to non-linear convergence theory. Most numerical schemes of electromagnetics do not offer direct control of the divergence of the discrete vector fields. However it is widely acknowledged that the success of edge elements is related to weak divergence preservation. In the simplest case the mechanism is as follows : Let $X_{h}$ denote the space of lowest order Nédélec edge elements on a simplicial triangulation with mesh-width $h$, and $Y_{h}$ denote the conditinuous piecewise affine functions on the same triangulation. The first standard remark is that (due to the tight connection between these FE spaces and simplicial cohomology), the cohomology group of the sequence :

$$
Y_{h} \stackrel{\operatorname{grad}}{\longrightarrow} X_{h} \stackrel{\text { curl }}{\longrightarrow} \mathrm{L}^{2},
$$

has the dimension of its continuous counterpart. This accounts for the naturality of considering $Y_{h}$ and $X_{h}$ together and has attracted much interest in the numerical analysis community, see Arnold [2]. Now in the Galerkin formulation of electromagnetic problems the only control available apriori on the divergence of an electric field $u_{h} \in X_{h}$ is in the form of the information :

$$
\left\{\left(p_{h}, \int u_{h} \cdot \operatorname{grad} p_{h}\right): p_{h} \in Y_{h}\right\} .
$$

How does this discrete divergence information translate into $\mathrm{H}^{-1}$ information on div $u_{h}$ ? For instance a basic question is : Suppose that we have a sequence $\left(\mathcal{T}_{h}\right)$ of shape-regular meshes with mesh-width $h \rightarrow 0$, yielding sequences of spaces $\left(X_{h}\right)$ and $\left(Y_{h}\right)$. Suppose that $\left(u_{h}\right)$ is a sequence of vector fields $u_{h} \in X_{h}$, bounded in $\mathrm{L}^{2}$ and discrete divergence free in the sense that :

$$
\forall p_{h} \in Y_{h} \quad \int u_{h} \cdot \operatorname{grad} p_{h}=0 .
$$

Is $\left(\operatorname{div} u_{h}\right)$ relatively compact in $\mathrm{H}^{-1}$ ?

I don't know the answer to this question. But an aim of this paper is to show a slightly weaker result namely Corollary 3.8 . This is a direct consequence of Theorem 3.7 which is a div-curl lemma for edge elements paralleling Theorem 1.1 .

\section{Setting}

Let $\Omega$ be a bounded domain in $\mathbb{R}^{3}$ whith a smooth boundary. For simplicity we suppose that $\Omega$ has the topological property that the kernel of the curl operator on $\mathrm{L}^{2}=\mathrm{L}^{2}(\Omega)$ is exactly the range of the gradient on $\mathrm{H}^{1}=\mathrm{H}^{1}(\Omega)$. The extension of the following results to general topology is straightforward using the fact that the edge elements approximate the $\mathrm{L}^{2}$ realisations of cohomology groups (harmonic vector fields) extremely well. Moreover the restriction on the regularity of the boundary is not too far-fetched since the property we are aiming at is essentially local. The standard norms and seminorms on $\mathbf{H}^{k}$ are denoted $\|\cdot\|_{k}$ and $|\cdot|_{k}$ respectively.

Let $X$ denote the space $\mathrm{L}^{2}, W$ the kernel of the curl $: X \rightarrow \mathrm{H}^{-1}$ and $V$ its $\mathrm{L}^{2}$ orthogonal. The elements of $V$ are divergence free and have vanishing normal component on $\partial \Omega$. 
Let $P_{V}$ denote the projection with range $V$ and kernel $W$. It is nothing but the $\mathrm{L}^{2}$ projection onto $V$ and it preserves the curl, i.e. for all vector fields $u \in \mathrm{L}^{2}:$

$$
\operatorname{curl} P_{V} u=\operatorname{curl} u \text {. }
$$

A less straightforward property of $V$ is the following :

Proposition 2.1 On $V,\|\operatorname{curl}(\cdot)\|_{-1}$ is a norm equivalent to the $\mathrm{L}^{2}$ norm.

-Proof: Theorem 3.20 (which requires $C^{1,1}$ regularity of the boundary) in Amrouche et al. [1] provides a closed subspace $U$ of $\mathbf{H}_{0}^{1}$ such that curl : $U \rightarrow V$ is an isomorphism. For any $v \in V$, putting $v=\operatorname{curl} u$ for $u \in U$ we have :

$$
\|\operatorname{curl} v\|_{-1}=\sup _{u^{\prime} \in \mathrm{H}_{0}^{1}}\left(\int \operatorname{curl} u \cdot \operatorname{curl} u^{\prime}\right) /\left\|u^{\prime}\right\|_{1} .
$$

and since the bilinear form :

$$
\left(u, u^{\prime}\right) \mapsto \int \operatorname{curl} u \cdot \operatorname{curl} u^{\prime},
$$

induces the norm on $U$, this completes the proof.

We will use the following setting: Let $\left(X_{h}\right)$ denote a sequence of finitedimensional subspaces of vector fields in $\mathrm{L}^{2}$, with curl in $\mathrm{L}^{2}$. The spaces $X_{h}$ are split into direct sums:

$$
X_{h}=V_{h} \oplus W_{h},
$$

such that $W_{h}$ is the kernel of the curl operator on $X_{h}$ and $V_{h}$ is its $\mathrm{L}^{2}$ orthogonal. Moreover we write:

$$
W_{h}=\operatorname{grad} Y_{h},
$$

where $Y_{h} \subset \mathrm{H}^{1}$ is a finite-dimensional space of functions with vanishing integral so that grad : $Y_{h} \rightarrow W_{h}$ is an isomorphism .

Some minimal properties are assumed throughout:

$$
\begin{aligned}
& \forall u \in \mathrm{L}^{2} \quad \lim _{h \rightarrow 0} \inf _{u_{h} \in X_{h}}\left\|u-u_{h}\right\|_{0}=0 . \\
& \forall p \in \mathrm{H}^{1} \quad \lim _{h \rightarrow 0} \inf _{p_{h} \in Y_{h}}\left\|p-p_{h}\right\|_{1}=0 .
\end{aligned}
$$

Of course all FE spaces satisfy them, and they can be interpreted as the pointwise convergence of the corresponding $\mathrm{L}^{2}$ and $\mathrm{H}^{1}$ projectors. These will be denoted $P_{h}^{0}$ and $P_{h}^{1}$.

One of the difficulties is that for the discretization spaces we are interested in, in general we have:

$$
V_{h} \not \subset V .
$$

However we shall use the fact that the elements of $V_{h}$ behave very much like those of $V$; precise statements are provided below.

In this setting we state the following assumptions :

DNE (Discrete norm equivalence) : There is $C>0$ such that:

$$
\forall h \forall v_{h} \in V_{h} \quad\left\|v_{h}\right\|_{0} \leq C\left\|\operatorname{curl} v_{h}\right\|_{-1} .
$$


SA (Super-approximation) : For any function $\phi \in \mathcal{D}$ (smooth and compactly supported) we have:

$$
\lim _{h \rightarrow 0} \sup _{p_{h} \in Y_{h}} \inf _{p_{h}^{\prime} \in Y_{h}}\left\|\phi p_{h}-p_{h}^{\prime}\right\|_{1} /\left\|p_{h}\right\|_{1}=0 .
$$

Both of them will not always be needed. I will therefore point out which ones are used for each statement of the paper. That the edge elements of Nédélec (adequately fitted near the curved boundary as in Dubois [17]), fullfill these assumptions is the object of the following propositions :

Proposition 2.2 Suppose the family of meshes $\left(\mathcal{T}_{h}\right)$ is quasi-uniform. Let $X_{h}$ be any edge element space constructed on the mesh $\mathcal{T}_{h}$. Then $\left(X_{h}\right)$ satifies (DNE).

As we shall see this is a very strong statement. For instance it is a considerable strengthening of Proposition 4.6 in [1], but has a more restrictive boundary regularity hypothesis.

-Proof: Let $\Pi_{h}$ denote the standard edge element interpolator. It maps curl free fields to curl free fields due to the commuting diagram property of standard interpolation operators. For any $v_{h}$ in $V_{h}, P_{V} v_{h}$ is in $\mathrm{H}^{1}$, due to our regularity assumptions on the boundary, and has a piecewise smooth curl. Therefore $\Pi_{h}$ is well defined on $P_{V} v_{h}$.

$$
\operatorname{curl}\left(\Pi_{h} P_{V} v_{h}-v_{h}\right)=\operatorname{curl} \Pi_{h}\left(P_{V} v_{h}-v_{h}\right)=0 .
$$

Hence $v_{h}, P_{V} v_{h}$ and $\Pi_{h} P_{V} v_{h}$ have the same curl. Next we use a trick due to V. Girault for which I refer to the proof of Lemma 4.1 in Ciarlet-Zou [14]. A Bramble-Hilbert type error estimate for $\Pi_{h}$, using the additional information available on curl $v_{h}$ (when transported from the cells of $\mathcal{T}_{h}$ to the reference cell, it lies in a fixed finite dimensional space) yields :

$$
\left\|\Pi_{h} P_{V} v_{h}-P_{V} v_{h}\right\|_{0} \leq C h\left|P_{V} v_{h}\right|_{1} .
$$

Then Lemma 2.11 in [1] gives :

$$
\left\|\Pi_{h} P_{V} v_{h}-P_{V} v_{h}\right\|_{0} \leq C h\left\|\operatorname{curl} v_{h}\right\|_{0},
$$

and we proceed as in the proof of Theorem 3.5 in [12]. Putting $w_{h}=\Pi_{h} P_{V} v_{h}-$ $v_{h} \in W_{h}$ we have :

$$
\left\|w_{h}\right\|_{0}^{2}=\int w_{h} \cdot\left(\Pi_{h} P_{V} v_{h}-P_{V} v_{h}\right) \leq\left\|w_{h}\right\|_{0}\left\|\Pi_{h} P_{V} v_{h}-P_{V} v_{h}\right\|_{0} .
$$

Hence :

$$
\left\|w_{h}\right\|_{0} \leq C h\left\|\operatorname{curl} v_{h}\right\|_{0} .
$$

Now using an inverse inequality we obtain:

$$
\begin{aligned}
\left\|P_{V} v_{h}-v_{h}\right\|_{0} & \leq\left\|\Pi_{h} P_{V} v_{h}-P_{V} v_{h}\right\|_{0}+\left\|\Pi_{h} P_{V} v_{h}-v_{h}\right\|_{0} \\
& \leq C h\left\|\operatorname{curl} v_{h}\right\|_{0} \\
& \leq C\left\|\operatorname{curl} v_{h}\right\|_{-1} .
\end{aligned}
$$

With this result and Proposition 2.1 one concludes. 
Proposition 2.3 Suppose the family of meshes $\left(\mathcal{T}_{h}\right)$ is quasi-uniform and pick an integer $k \geq 1$. Let $Y_{h}$ be the space of continuous piecewise polynomials of degree less that $k$, constructed on the mesh $\mathcal{T}_{h}$. Then $\left(Y_{h}\right)$ satifies (SA).

-Proof: In fact much more precise estimates are known. See Wahlbin [36] p. 36-37 for a discussion. In particular for $k=1$ the quasi-uniformity hypothesis is not necessary, and the proposition follows from the Bramble-Hilbert lemma remarking that if $\Pi_{h}$ is the standard nodal interpolator :

$$
\forall p_{h} \in Y_{h} \quad\left\|\Pi_{h}\left(\phi p_{h}\right)-\phi p_{h}\right\|_{1} \leq C h \sum_{T}\left|\phi p_{h}\right|_{T, 2} \leq C h\left\|p_{h}\right\|_{1},
$$

since the second order derivatives of $p_{h}$ vanish.

\section{Results}

\subsection{On weak convergence}

Lemma 3.1 Suppose $u_{h} \in X_{h}$ converges weakly in $\mathrm{L}^{2}$, to $u=v+w$ with $v \in V$ and $w \in W$. Then with the decomposition $u_{h}=v_{h}+w_{h}, v_{h} \in V_{h}$ and $w_{h} \in W_{h}$, $\left(v_{h}\right)$ and $\left(w_{h}\right)$ converge weakly to $v$ and $w$ in $\mathrm{L}^{2}$.

-Proof: Put $w_{h}=\operatorname{grad} p_{h}$ with $p_{h} \in Y_{h}$. Let $P_{h}$ denote the Galerkin projection onto $Y_{h}$ with respect to the bilinear form :

$$
\left(p, p^{\prime}\right) \rightarrow \operatorname{grad} p \cdot \operatorname{grad} p^{\prime}
$$

Then we have, for all $p \in \mathrm{H}^{1}(\Omega)$ :

$$
\int \operatorname{grad} p_{h} \cdot \operatorname{grad} p=\int \operatorname{grad} p_{h} \cdot \operatorname{grad} P_{h} p=\int u_{h} \cdot \operatorname{grad} P_{h} p .
$$

Since $\left(P_{h} p\right)$ converges strongly to $p$ in $\mathrm{H}^{1}$, the above quantity converges to :

$$
\int u \cdot \operatorname{grad} p=\int w \cdot \operatorname{grad} p .
$$

It follows that $\left(w_{h}\right)$ converges weakly in $\mathrm{L}^{2}$ to $w$. Therefore $\left(v_{h}\right)$ also converges weakly to $v$.

\subsection{On relatively compact sequences}

The following result uses (DNE).

Proposition 3.2 Let $\left(v_{h}\right)$ be a sequence of vector fields such that $v_{h} \in V_{h}$.

- If ( $\left.\operatorname{curl} v_{h}\right)$ converges to curl $v$ in $\mathrm{H}^{-1}$ for some $v \in V$, then $\left(v_{h}\right)$ converges to $v$ in $\mathrm{L}^{2}$.

- If $\left(\operatorname{curl} v_{h}\right)$ is relatively compact in $\mathrm{H}^{-1}$, then $\left(v_{h}\right)$ is relatively compact in $\mathrm{L}^{2}$. 
-Proof : By Proposition 2.1, $\left(P_{V} v_{h}\right)$ converges to $v$ in $\mathrm{L}^{2}$. Also, if we let $P_{h}$ denote the $\mathrm{L}^{2}$ projection onto $X_{h},\left(P_{h} v\right)$ converges to $v$ in $\mathrm{L}^{2}$. We now write :

$$
\begin{aligned}
\left\|P_{h} v-v_{h}\right\|_{0} & \leq C\left\|\operatorname{curl} P_{h} v-\operatorname{curl} v_{h}\right\|_{-1} \\
& \leq C\left\|\operatorname{curl} P_{h} v-\operatorname{curl} P_{V} v_{h}\right\|_{-1} \\
& \leq C\left\|P_{h} v-P_{V} v_{h}\right\|_{0}
\end{aligned}
$$

It follows that $\left(v_{h}\right)$ converges to $v$.

The second point of the proposition follows from the first one by the characterization of relative compactness in terms of extracting convergent subsequences out of subsequences.

\subsection{On discrete compactness}

The following results use (DNE).

This section is not used in the rest of this article and is included just to show the relationship between the (DNE), the gap property of discrete Helmholtz or Hodge decompositions which proved useful in analysing some integral equations describing electromagnetic phenomena (Christiansen [12] and Buffa-Christiansen [10]) and the discrete compactness property of edge elements in the sense of Kikuchi [24] which is a crucial tool in analysing the discrete eigenvalue problem of electromagnetics (see in particular Boffi et al. [4][5]).

Suppose $Z$ is a Hilbert space such that $\mathrm{L}^{2} \subset Z \subset \mathrm{H}^{-1}$ with continuous inclusions. Suppose furthermore that the injection $Z \rightarrow \mathrm{H}^{-1}$ is compact.

Proposition 3.3 We have :

$$
\lim _{h \rightarrow 0} \sup _{v_{h} \in V_{h}}\left\|v_{h}-P_{V} v_{h}\right\|_{0} /\left\|\operatorname{curl} v_{h}\right\|_{Z}=0 .
$$

-Proof: Indeed if this were not true we would have a subsequence $\left(v_{h}\right)$ with $v_{h} \in V_{h}$ such that $\left(\left\|\operatorname{curl} v_{h}\right\|_{z}\right)$ is bounded and $\left(\left\|v_{h}-P_{V} v_{h}\right\|_{0}\right)$ tends to infinity. From it we can extract a subsequence such that (curl $v_{h}$ ) converges weakly in $Z$. But then (curl $v_{h}$ ) converges strongly in $\mathrm{H}^{-1}$. One obtains a contradiction with Proposition 3.2.

Consider the Hilbert space $X_{Z}$ of vector fields in $\mathrm{L}^{2}$ with curl in $Z$. In this space, equipped with its natural norm $\|\cdot\|_{0}$, let $V_{Z}$ be the $\mathrm{L}^{2}$ orthogonal of (the closed subspace) $W=\operatorname{grad} \mathrm{H}^{1}$ and let $\delta_{Z}(\cdot, \cdot)$ denote the gap between supspaces in the sense of Kato [23].

Corollary 3.4 We have:

$$
\lim _{h \rightarrow 0} \delta_{Z}\left(V_{h}, V_{Z}\right)=0
$$

-Proof : Just remark that :

$$
\left\|v_{h}-P_{V} v_{h}\right\|_{0 Z}=\left\|v_{h}-P_{V} v_{h}\right\|_{0} \quad \text { and } \quad\left\|\operatorname{curl} v_{h}\right\|_{Z} \leq\left\|v_{h}\right\|_{0 Z}
$$

and conclude using Proposition 3.3.

From this gap property in the case $Z=\mathrm{L}^{2}$ one easily deduces the following discrete compactness property. Let $X_{0}$ denote the space of vector fields $u$ in $\mathrm{L}^{2}$, such that curl $u$ is in $\mathrm{L}^{2}$. Let $V_{0}$ denote the $\mathrm{L}^{2}$ orthogonal of the closed subspace 
$W=\operatorname{grad} \mathrm{H}^{1}$ of $X_{0}$. The elements of $V_{0}$ thus have vanishing divergence and vanishing normal component on $\partial \Omega$. By a result of Weber for which I refer to Theorem 2.8 in [1], $V_{0}$ is compactly imbedded in $\mathrm{L}^{2}$. This result has the following discrete analogue :

Corollary 3.5 Suppose $\left(v_{h}\right)$ is a bounded sequence of vector fields in $X_{0}$, such that $v_{h} \in V_{h}$. Then there is a subsequence which converges in $\mathrm{L}^{2}$.

-Proof: If $\left(v_{h}\right)$ is such a sequence, then $\left(P_{V} v_{h}\right)$ is bounded in $V_{0}$ and $\left(\| v_{h}-\right.$ $\left.P_{V} v_{h} \|_{0}\right)$ tends to 0 .

\subsection{Div-Curl lemma for edge elements}

Lemma 3.6 uses (SA), Theorem 3.7 and Corollary 3.8 use both (DNE) and (SA).

Lemma 3.6 Suppose $\left(v_{h}\right)$ is a sequence of vector fields $v_{h} \in V_{h}$ converging weakly in $\mathrm{L}^{2}$ to $v$, and $p_{h}$ is a sequence of scalar fields $p_{h} \in Y_{h}$ converging weakly in $\mathrm{H}^{1}$ to $p$. Then $\left(v_{h} \cdot \operatorname{grad} p_{h}\right)$ converges vaguely to $v \cdot \operatorname{grad} p$ in $\mathcal{D}^{\prime}$.

-Proof: Pick $\phi \in \mathcal{D}$. We have :

$$
\int\left(v_{h} \cdot \operatorname{grad} p_{h}\right) \phi=\int v_{h} \cdot \operatorname{grad}\left(\phi p_{h}\right)-\int\left(v_{h} \cdot \operatorname{grad} \phi\right) p_{h} .
$$

Since $v_{h} \in V_{h}$, the first term can be replaced by :

$$
\int v_{h} \cdot \operatorname{grad}\left(\phi p_{h}-P_{h}^{1}\left(\phi p_{h}\right)\right)
$$

It converges to 0 by (SA).

In the second term we remark that $\left(v_{h} \cdot \operatorname{grad} \phi\right)$ converges weakly to $v \cdot \operatorname{grad} \phi$ in $\mathrm{L}^{2}$, whereas $\left(p_{h}\right)$ converges strongly in $\mathrm{L}^{2}$.

Therefore :

$$
\int\left(v_{h} \cdot \operatorname{grad} p_{h}\right) \phi \rightarrow-\int(v \cdot \operatorname{grad} \phi) p=\int(v \cdot \operatorname{grad} p) \phi .
$$

This completes the proof.

Theorem 3.7 Suppose $\left(u_{h}\right)$ and $\left(u_{h}^{\prime}\right)$ are sequences of vector fields $u_{h}, u_{h}^{\prime} \in$ $X_{h}$ converging weakly in $\mathrm{L}^{2}$ to $u$ and $u^{\prime}$. Suppose furthermore that with the decomposition $u_{h}=v_{h}+\operatorname{grad} p_{h}$ for $v_{h} \in V_{h}$ and $p_{h} \in Y_{h},\left(p_{h}\right)$ is relatively compact in $\mathrm{H}^{1}$, and that (curl $u_{h}^{\prime}$ ) is relatively compact in $\mathrm{H}^{-1}$.

Then $\left(u_{h} \cdot u_{h}^{\prime}\right)$ converges vaguely to $u \cdot u^{\prime}$ in $\mathcal{D}^{\prime}$.

-Proof: Decompose also $u_{h}^{\prime}=v_{h}^{\prime}+\operatorname{grad} p_{h}^{\prime}$ with $v_{h}^{\prime} \in V_{h}$. Recall that by Lemma 3.1 all terms of the decompositions converge weakly. The limits will be denoted by skipping the subscipt $h$.

Pick $\phi \in \mathcal{D}$. Now $\int u_{h} \cdot u_{h}^{\prime} \phi$ can be decomposed into four terms :

For the term :

$$
\int\left(\operatorname{grad} p_{h} \cdot \operatorname{grad} p_{h}^{\prime}\right) \phi
$$


we remark that $\left(\operatorname{grad} p_{h}\right)$ converges strongly in $\mathrm{L}^{2}$ by the hypothesis. This term therefore converges to :

$$
\int\left(\operatorname{grad} p \cdot \operatorname{grad} p^{\prime}\right) \phi,
$$

For the term :

$$
\int\left(v_{h} \cdot v_{h}^{\prime}\right) \phi,
$$

we remark that by Proposition $3.2, v_{h}^{\prime}$ converges strongly in $\mathrm{L}^{2}$ to $v$. Hence this term converges to

$$
\int\left(v \cdot v^{\prime}\right) \phi,
$$

For the cross terms, one is trivial and the other is covered by Lemma 3.6, and this completes the proof.

A direct and useful consequence of Theorem 3.7 is :

Corollary 3.8 Suppose $\left(u_{h}\right)$ and $\left(u_{h}^{\prime}\right)$ are sequences of vector fields $u_{h}, u_{h}^{\prime} \in X_{h}$ converging weakly in $\mathrm{L}^{2}$ to $u$ and $u^{\prime}$. Suppose furthermore that $u_{h}$ is discrete divergence free (i.e. is in $V_{h}$ ) and that ( $\left.\operatorname{curl} u_{h}^{\prime}\right)$ is bounded in $\mathrm{L}^{2}$.

Then $\left(u_{h} \cdot u_{h}^{\prime}\right)$ converges vaguely to $u \cdot u^{\prime}$ in $\mathcal{D}^{\prime}$.

The next remark shows that on one point the hypothesis of Theorem 3.7 is not strictly stronger than that of Theorem 1.1:

Remark 3.9 Suppose $\left(u_{h}\right)$ is a sequence of vector fields $u_{h} \in X_{h}$ such that ( $\operatorname{div} u_{h}$ ) is relatively compact in $\mathrm{H}^{-1}$. Then with the decomposition $u_{h}=v_{h}+$ grad $p_{h}$, for $v_{h} \in V_{h}$ and $p_{h} \in Y_{h},\left(p_{h}\right)$ is relatively compact in $\mathrm{H}^{1}$.

-Proof: This follows from the uniform continuity and pointwise convergence in $\mathrm{H}^{1}$ of the Galerkin projectors $\left(P_{h}\right)$ introduced in the proof of Lemma 3.1.

\section{Discussion}

I close this paper with some remarks.

Of course, if an entire physical field is to be approximated numerically, a scheme which is only vaguely converging is of little practical use. Indeed the consensus seems to be to aim for rapid convergence in the energy norm. However a method which satisfies a given property (vague convergence for instance) under the weakest of assumptions shows a sign of robustness, in the sense that under extreme conditions it performs as well as one can reasonably hope for. Therefore the above result can be interpreted as a robustness property of edge elements with respect to a class of nonlinearities.

It is sometimes useful to state properties of discretized electromagnetic fields in terms of (non-smooth) differentiable forms (see Hiptmair [20] for more on this). Likewise the div-curl lemma of Murat Tartar can be stated in terms of differential forms (see Taylor [35] p. 358-359). The translation of the results of this paper into this framework is unproblematic. A case of particular interest is when considering div conforming face elements [31][9]. One obtains a similar div-curl lemma for face-elements based this time on the discrete information on the curl (obtained by integrating against the curl of the naturally associated edge element space). 
The question whether $\mathrm{L}^{2}$ bounded sequences in $V_{h}$ (discrete vector fields which are discrete divergence free) have relatively compact divergence in $\mathrm{H}^{-1}$ is as of today unsettled for the standard FE spaces, and as mentioned in the introduction the present paper can be seen as a circumvention of this question. The affirmative would imply a slightly stronger version of Theorem 3.7 (less restrictions on $\left.\left(u_{h}^{\prime}\right)\right)$.

The div-curl lemma is closely related to Hardy space estimates (see e.g. Coifman et al. [15]) and a translation of the above results into uniform Hardy space estimates for edge elements would be an important step forward.

Since much is known a priori about FE spaces and discrete schemes one might muse at the possibility that an existence theorem for a hard non-linear problem may one day be proved by studying the convergence of a sequence of FE solutions.

\section{Acknowledgements}

This work is inspired by a joint effort to understand the discretization of some non-linear equations [13].

\section{References}

[1] C. Amrouche, C. Bernardi, M. Dauge, V. Girault : Vector potentials in three-dimensional non-smooth domains ; Math. Meth. Appl. Sci., Vol. 21, No. 9, p. 823-864, 1998.

[2] D.N. ARnold : Differential complexes and numerical stability ; Plenary address delivered at ICM 2002, International Congress of Mathematicians, Beijing 2002 .

[3] I. Babuska : Error bounds for the finite element method ; Numer. Math., Vol. 16, p. 322-333, 1971.

[4] D. Boffi, F. Brezzi, L. Gastaldi : On the problem of spurious eigenvalues in the approximation of linear elliptic problems in mixed form; Math. Comp., Vol. 69, No. 229, p. 121-140, 1999.

[5] D. Boffi, P. Fernandes, L. Gastaldi, I. Perugia : Computational models of electromagnetic resonators: analysis of edge element approximation ; SIAM J. Numer. Anal., Vol. 36, No. 4, p. 1264-1290, 1999.

[6] D. Boffi : A note on the DeRham complex and a discrete compactness property; Appl. Math. Lett., Vol. 14, No. 1, p. 33-38, 2001.

[7] A. Bossavit : Mixed finite elements and the complex of Whitney forms ; The mathematics of finite elements and applications, VI (Uxbridge, 1987), p. 137-144, Academic Press, London, 1988.

[8] F. BREzzI : On the existence, uniqueness and approximation of saddlepoint problems arising from Lagrangian multipliers ; RAIRO Anal. Numér., Vol. 8, No. R-2, p. 129-151, 1974. 
[9] F. Brezzi, M. Fortin : Mixed and hybrid finite element methods ; Springer-Verlag, 1991.

[10] A. Buffa, S. H. Christiansen : The Electric Field Integral Equation on Lipschitz screens : definitions and numerical approximation; Numer. Math., Vol. 94, No. 2, p. 229-267, 2003.

[11] M. Cessenat : Mathematical methods in electromagnetism, Linear theory and applications; World Scientific Publishing Co., River Edge, NJ, 1996.

[12] S. H. Christiansen : Discrete Fredholm properties and convergence estimates for the Electric Field Integral Equation ; Math. Comp., to appear, PII : S 0025-5718(03)01581-3.

[13] S. H. Christiansen, G. Ellingsrud, R. Kozlov, R. Winther : Numerical aspects of constraint preservation in Yang-Mills equations ; work in progress.

[14] P. Ciarlet Jr., J. Zou : Fully discrete finite element approaches for time-dependant Maxwell's equations ; Numer. Math., Vol. 82, p. 193-219, 1999 .

[15] R. Coifman, P.-L. Lions, Y. Meyer, S. Semmes : Compensated compactness and Hardy spaces; J. Math. Pures Appl (9), Vol. 72, No. 3, p. 247-286, 1993.

[16] M. Costabel, M. Dauge : Singularities of electromagnetic fields in polyhedral domains ; Arc. Rat. Mech. and Anal., Vol. 151, No. 3, p. 221-276, 2000 .

[17] F. Dubois : Discrete vector potential representation of a divergence free vector field in three dimensional domains : Numerical analysis of a model problem ; SIAM J. Numer. Anal., Vol. 27, p. 1103-1142, 1990.

[18] G. B. Folland : Real Analysis : Modern techniques and their applications, Second Edition; John Wiley \& Sons, 1999.

[19] V. GiRault : Incompressible finite element methods for Navier-Stokes equations with nonstandard boundary conditions in $\mathbb{R}^{3} ;$ Math. Comp., Vol. 51, p. 55-74, 1988.

[20] R. Hiptmain : Finite elements in computational electromagnetism ; Acta Numerica, p. 237-339, Cambridge University Press, 2002.

[21] J.D. Jackson : Classical electrodynamics, Second edition; John Wiley \& Sons, New York-London-Sydney, 1975.

[22] P. Joly : Variational methods for time-dependent wave propagation problems ; course notes, INRIA Rocquencourt, BP105 Le Chesnay, France, 2002 .

[23] T. Kato : Perturbation theory for linear operators, Second edition ; Springer-Verlag, Berlin-New York 1976. 
[24] F. KIKUCHI : On a discrete compactness property for the Nédélec finite elements ; J. Fac. Sci. Univ. Tokyo, Sect. 1A Math., Vol. 36, p. 479-490, 1989 .

[25] P. Monk : Finite Element Methods for Maxwell's Equations ; Oxford University Press, 2003.

[26] F. Murat : Compacité par compensation ; Ann. Scuola Norm. Sup. Pisa (4), Vol. 5, No. 3, p. 485-507, 1978.

[27] J. NEČAS : Les méthodes directes en théorie des équations elliptiques ; Masson, Paris, 1967.

[28] J.-C. NÉDÉLEC : Mixed finite elements in $\mathbb{R}^{3}$; Numer. Math., Vol. 35, p. 315-341, 1980.

[29] J.-C. NÉDÉLEC : A new family of mixed finite elements in $\mathbb{R}^{3}$; Numer. Math., Vol. 50, No. 1, p. 57-81, 1986.

[30] J.-C. NÉDÉLEC : Acoustic and electromagnetic equations, Integral representations for harmonic problems ; Springer-Verlag, 2001.

[31] P.A. Raviart, J.-M. Thomas : A mixed finite element method for 2nd order elliptic problems ; p. 292-315 in I. Galligani, E. Magenes (ed): Mathematical aspects of the finite element method ; Lecture Notes in Math., Vol. 606, Springer-Verlag, Berlin and New York, 1977.

[32] G. DeRham : Variétés différentiables. Formes, courants, formes harmoniques (Third edition) ; Publications de l'Institut de Mathématique de l'Université de Nancago, III. Actualités Scientifiques et Industrielles, No. 1222b. Hermann, Paris, 1973.

[33] W. Rudin : Functional Analysis, Second Edition ; McGraw-Hill, New York, 1991.

[34] L. TARTAR : Compensated compactness and applications to partial differential equations ; in "Nonlinear analysis and mechanics: Heriot-Watt Symposium”, Vol. IV, p. 136-212, Res. Notes in Math., Vol. 39, Pitman, Boston, Mass.-London, 1979.

[35] M. TaYlor : Partial differential equations, Vol. I Basic theory; SpringerVerlag, New York, 1996.

[36] L. B. Wahlbin : Superconvergence in Galerkin finite element methods ; LNM 165, Springer-Verlag, 1995.

[37] H. Whitney : Geometric integration theory ; Princeton University Press, Princeton, N. J., 1957. 